\title{
Introduction on the Emotion-Will Overcoming Therapy (EWOT): A Novel Alternative Approach of Psychological Treatment from Chinese Medicine
}

\author{
Hui Zhang1, Xiangeng Zhang1* Xiaoli Liang1, Han Lai², Jin Gao1, Qin Liu1, Hongyan Wang1 \\ ${ }^{1}$ The School of Nursing, Chengdu University of TCM, Chengdu, China \\ ${ }^{2}$ The School of Foreign Language, Chengdu University of TCM, Chengdu, China \\ Email: ${ }^{*}$ jeffery.h.zhang@gmail.com
}

Received 17 March 2015; accepted 7 May 2015; published 12 May 2015

Copyright $@ 2015$ by authors and Scientific Research Publishing Inc.

This work is licensed under the Creative Commons Attribution International License (CC BY). http://creativecommons.org/licenses/by/4.0/

(c) (i) Open Access

\begin{abstract}
Emotional disorders and mental illnesses constitute a significant part of diseases. In Chinese medicine, emotional disorders and mental illnesses are classified as emotion-will (Qing Zhi) disorders. Emotion-will, i.e. seven emotions ( $Q$ i Qing: happiness, anger, anxiety, pensiveness, sorrow, fear, and fright) and five wills ( $W u$ Zhi: happiness, anger, thinking, sorrow, and fear), play a basic role in the onset, progress and prognosis of almost all diseases, not only the mental illnesses. The emotion-will overcoming therapy (EWOT) is defined as a psychological approach that a therapist employs single or multiple emotions to overcome and eliminate patients' abnormal morbid emotions and to heal mind-body disorders derived from the abnormality. EWOT lays a foundation for the philosophical foundation of Chinese medicine, i.e. Yin and Yang, and five elements, which is believed to be the most commonly utilized and effective modality in dealing with mental illnesses. This essay covers the origin and development, underlying mechanism, clinical application, basic researches of emotion-will system and EWOT and the comparison with conventional therapies. Thus, EWOT could draw more attention in the field of psychology and spread in clinical practice.
\end{abstract}

\section{Keywords}

CM Psychology, Emotion-Will Overcoming Therapy, Psychology, Seven Emotions, Five Wills

\footnotetext{
*Corresponding author.
}

How to cite this paper: Zhang, H., et al. (2015) Introduction on the Emotion-Will Overcoming Therapy (EWOT): A Novel Alternative Approach of Psychological Treatment from Chinese Medicine. Chinese Medicine, 6, 75-82. 


\section{Introduction}

The theory of emotion and will refers to the seven emotions (Qi Qing: happiness, anger, worry, thinking, sorrow, fear, and fright) and five wills ( $W u$ Zhi: happiness, anger, thinking, sorrow, and fear) [1]. Hereby, "will" means normal emotional activities and "emotion" means hyperactive activities. In Chinese medicine, emotion-will plays a basic role in the onset, progress, and prognosis of almost all diseases, not only the psychological and psychiatric disorders. The emotion-will overcoming therapy (EWOT) derived from the philosophical foundation of Chinese medicine, i.e. Yin and Yang, and five elements, is believed to be the most effective modality in dealing with mental illness.

For thousands years, medical experts have been exploring the importance of emotion-will and efficacy of EWOT in medical records since the establishment of Chinese medicine theoretic and therapeutic structure in Yellow Emperor's Inner Classic (Huang Di Nei Jing) [2]. At present, Chinese medicine practitioners still frequently utilize EWOT in treating depression, anxiety, phobia, etc. and nursing chronic diseases such as apoplexy sequelae, cancer, diabetes, irritable bowel movement and so on [3]-[6]. From the 1980s on, Chinese researchers have been trying to translate this emotion-will with very Chinese characteristic in conventional psychological language. Some promising results have been made. For instance, relationships and similarities among EWOT and cognitive and behavior therapy (CBT) [7], positive psychology therapy [8], and Morita therapy (MT) [9] [10] have been discussed. But, regrettably the emotion-will system and EWOT have not been introduced to the Chinese medicine practitioners and psychology therapists outside China except for rare literature with rough description [11] [12]. Comparing with the prosperous spread of acupuncture and herbal medicine, two major therapy approaches in Chinese medicine, EWOT is, however, a brand-new option and promising method dealing with mental illness [4] [13] [14]. This essay covers the origin and development, underlying mechanism, clinical application, basic researches of emotion-ill system and EWOT and the comparison with conventional therapies.

\section{Origin and Development of Emotion-Will and EWOT}

Records on emotion-will date back to The Book of Rites (Li Ji, 51-21 A.D.), in which happiness, anger, sorrow, fear, love, hate, and desire, the seven emotional reactions human beings have [15]. It is in Yellow Emperor's Inner Classic that the theoretic foundation is established. This book elaborates the relationship between emotion-will and organs, the pathogenesis of illness resulting from extreme emotions, and the treatment of emotional disorders, leading to a sound foundation for the development of the emotion-will theory.

The relationship is constructed on the model of five organ and five wills and emotions linked by the five elements theory, that are, liver-anger, heart-happiness, lungs-grief, spleen-pensiveness, kidneys-fear (Figure 1).

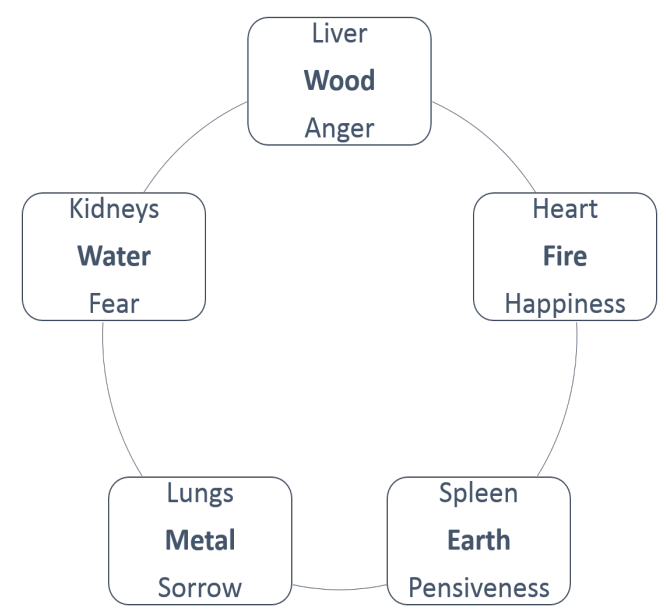

Figure 1. Organ-will pattern in understanding the correlation between five will and five organs in Chinese medicine and Chinese medicine psychology. A will and an organ, e.g. anger and liver are closely related because they have attributes of the element wood. Wood is featured by bending and straightening and likes orderly reaching. Anger drives the $Q i$ circulation. Liver is the organ that governs free flow of $Q i$. Based on the attribute similarity of liver, anger-wood, organ-will pattern is established and so does happiness-heart, pensiveness-spleen, sorrow-lungs, and fear-kidneys by an extension of this logic. 
This principle is considered as the foundation of emotion-will therapy in the treatment of emotional disorders and mental illnesses followed by later Chinese medicine practitioners supplementing and developing it. There are three representative advancements. First, Dr. Wuze Chen (1131-1189 A.D.) clarified the seven emotions, i.e. Qi Qing: happiness, anger, worry, thinking, sorrow, fear, and fright and differentiated the connotations of the Seven Emotions and five wills. Second, Congzheng Zhang (1156-1228 A.D.) recorded plenty of medical cases covering the utilization of EWOT in treating emotion-will disorders. Third, Wan Su Liu innovated a new understanding of extreme emotions, that is, a hyperactive emotion which gives rise to internal fire and accordingly injuries the related organ. In the 1980s, Prof. Miqu Wang and his peers believed in the modernization and systematization of the emotion-will theory. Thus Chinese medicine psychology (CMP), a new discipline, emerged, which bases on the philosophic essences of Chinese medicine and borrows the structure from current conventional psychology [16]. Still, in Chinese medicine psychology EWOT is a major approach.

\section{Mechanism of EWOT}

\subsection{Five Elements}

EWOT is defined as a psychological approach in which a therapist employs single or multiple emotions to overcome or eliminate patients' morbid emotions and to heal mind-body disorder derived from the abnormality. Speaking of the mechanism, pathogenesis and phatho-mechanism are the fundamental contents. From the perspective of pathogenesis, extreme performance of the five emotions engenders illness. When the will-emotion or psychological reaction manifests within the limits of rational range, it is the reflection of the normal function of internal organs i.e. "five will". A normal will benefit the $Q i$ transformation or the function of intern organs, health, and longevity. Once an emotion-will presents abnormally (insufficiently and excessively), most excessively, it results in diverse illness, i.e. "Seven Emotion".

As for the patho-mechanism (Bing $J i$ ), the injury of organs from the abnormality of emotion-will corresponds with the principle of the five elements, that is, hyper-anger harms liver, hyper happiness harms heart, hyperpensiveness harms spleen, hyper-sorrow harms lungs, and hyper-fear harms kidneys (Figure 2). It is the abnormality of emotion-will that arouses the disorder of $Q i$ circulation which is believed to be the primary reason for all diseases. According to Yellow Emperor's Inner Classic, anger drives $Q i$ upward, happiness causes $Q i$ to slacken, sorrow dissipates $Q i$, fear drives $Q i$ downward, pensiveness causes $Q i$ to bind, fright causes disruption of $Q i$ (Figure 3). In terms of the treatment of emotion-will disorder, its theoretic foundation is laid on the over

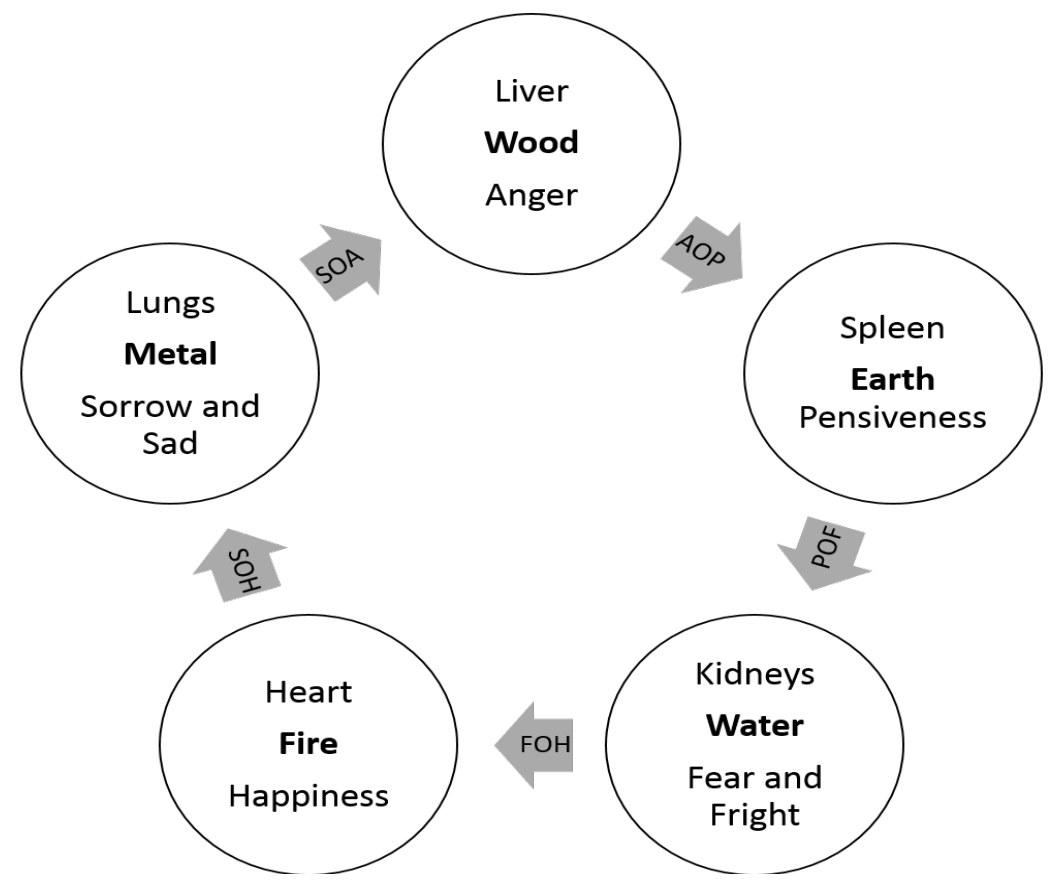

Figure 2. Emotion-will overcoming therapy (EWOT) model based on five elements. 


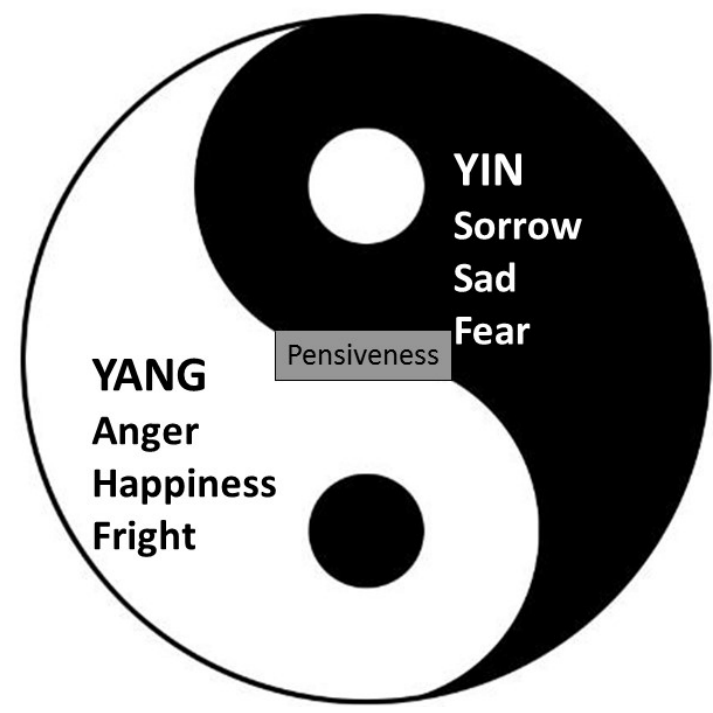

Figure 3. Emotion-will overcoming therapy (EWOT) model based on Yin and Yang. Yin can overcome hyperactive Yang and vice versa. Thus clinically, patient with emotional abnormalities of Yang (happiness, anger, and fright), are treated with emotions of Yin (sorrow, sad, and fear), while patient with emotional abnormalities of Yin (sorrow, sad, and fear), are treated with emotions of Yang (happiness, anger, and fright). In this way, the imbalance of Yin and Yang is restored, which indicates a mental illness is healed.

coming relationship among the five elements. It is constituted from five techniques: 1) sorrow overcomes the anger (SOA), 2) fear overcomes the happiness (FOH), 3) anger overcomes the pensiveness (AOP), 4) happiness overcomes the sorrow and sadness (HOSS), and 5) pensiveness overcomes the fear and fright (POFF) (Figure 2).

SOA: Sorrow attributes to the element metal and anger to the element wood. Because metal retrains wood, rational anger (one of the five wills) can overcome/neutralize hyperactive anger (one of the seven emotions).

FOH: Fear attributes to the element water and happiness to the element fire. Because water retrains fire, rational fear (one of the five wills) can overcome/neutralize hyperactive happiness (one of the seven emotions).

AOP: Anger attributes to the element wood and pensiveness to the element earth. Because wood retrains earth, rational anger (one of the five wills) can overcome/neutralize hyperactive pensiveness (one of the seven emotions).

HOSS: Happiness attributes to the element fire and sorrow to the element metal. Because fire retrains metal, rational happiness (one of the five wills) can overcome/neutralize hyperactive sorrow and sadness (two emotions of the seven emotions).

POFF: Pensiveness attributes to the element earth and fear to the element water. Because earth retrains water, rational pensiveness (one of the five wills) can overcome/neutralize hyperactive fear and fright (two emotions of the seven emotions).

SOA: Sorrow attributes to the element metal and anger to the element wood. Because metal retrains wood, rational anger (one of the five wills) can overcome hyperactive anger (one of the seven emotions).

FOH: Fear attributes to the element water and happiness to the element fire. Because water retrains fire, rational fear can overcome/neutralize hyperactive happiness.

AOP: Anger attributes to the element wood and pensiveness to the element earth. Because wood retrains earth, rational anger can overcome/neutralize hyperactive pensiveness.

HOSS: Happiness attributes to the element fire and sorrow to the element metal. Because fire retrains metal, rational happiness can overcome/neutralize hyperactive sorrow and sadness.

POFF: Pensiveness attributes to the element earth and fear to the element water. Because earth retrains water, 
rational pensiveness can overcome hyperactive fear and fright.

\subsection{Role of Yin and Yang}

Besides the Five Elements, Yin and Yang serves as another philosophical foundation in every aspects of Chinese medicine, from pathogenesis to treatment. Diverse discussions on the role the Yin and Yang plays can be found in Yellow Emperor's Inner Classic. For instance, when Yang is excessive, happiness presents; when Yin is excessive, anger presents (Plain Questions, On Manipulating Needles (Su Wen, Xing Zhen)). According to the attributes of Yin and Yang, Seven Emotions are categorized into two groups. First, happiness, anger, and fright attribute to Yang because the three emotions promote the circulation of $Q i$ and blood. Second, sorrow, sad, and fear to Yin, which slow down the circulation. Pensiveness is a unique and neutralized emotion with both Yin and Yang's qualities. As Yin can overcome hyperactive Yang and vice versa. Thus clinically, patient with emotional abnormalities of Yang (happiness, anger, fright), are treated with emotions of Yin (sorrow, sad, fear), while patient with emotional abnormalities of Yin (sorrow, sad, fear), are treated with emotions of Yang (happiness, anger, fright). In this way, the dis-balance of Yin and Yang is restored which is to say a mental illness is healed (Figure 3).

\section{Clinical and Basic Research on EWOT}

EWOT is regarded as a major clinical non-drug intervention in Chinese medicine psychology. For thousands years, a large amount of medical cases have been kept. Wang et al. [17] analyzed 196 ancient cases of disorders induced by seven emotion and found that EWOT was the most commonly applied approach. Yang et al. [18] analyzed 122 cases and found similar results as Wang's report. Meanwhile, other Chinese medicine psychology therapies were also applied such as suggestion therapy, stimulation therapy, behavior conduction therapy, etc., which were less frequently used than EWOT. Liu [19] (2009) treated 12 college students suffering phobia only with the technique of POF, resulting in satisfactory outcome. Clinically, EWOT is not only used to treat emotional disorders or mental illness, but also to treat and nurse other diseases e.g. menopause syndrome, irritable bowel syndrome, cardiovascular illnesses [20]-[22]. Ye et al. [23] found EWOT could alleviate anxiety and depression of stroke patients and enhanced their enthusiasm and initiative to take part in rehabilitation. Chen [24] reported a positive role EWOT played in the treatment of asthma, supported by the improved quality of life.

Current researches on the foundation are concentrated upon the sorting and editing ancient literature and experiments on the exploration of how the seven emotions impairs organ. Liu et al. [25] found the experimental evidence that prenatal fear derived from earthquake mimic could cause developmental insufficiency manifested as slower growth and poorer behavior performances. The finding is a convincing support for the understanding of EWOT. Zhang et al. [26] found that maternal fear negatively affected physical and nervous system development of the fetus, with specific alterations in neuro-hormones and gene expression. Interestingly, JKSQW (Jin Kui Shen $Q i$ Wan) a preventative Chinese herbal formula reduced these negative outcomes which proved that the kidneys' function was disturbed by fear from another angel. Although plenty of clinical reports on EWOT are available, unfortunately, there are no reports available on fundamental researches of the underlying mechanism of the five techniques in EWOT.

\section{EWOT and Conventional Psychology}

EWOT plays an essential role in Chinese medicine psychology. It has a distinctive feature of Chinese culture. Recently, researchers have paid attention to the comparative study for it with conventional western psychology therapies. Yan et al. [27] found the application of EWOT in the treatment of emotional disorders and mental illnesses was in accordance with some therapies in conventional psychology, for instance, supportive therapy, behavior therapy, cognitive therapy, etc. Huang [8] believed that the technique HOS is pretty similar to positive psychology created by Martin E. P. Seligam. Positive psychology interventions (PPIs) is a promising approach to increase happiness and well-being, that is, treatment methods or intentional activities that aim to cultivate positive feelings, behaviors, or cognitions [28]. With intervention of HOS, approaches making a patient happier e.g. joyful music, conversation, favorite foods, reading and so on are employed to overcome the sorrow the patient encounters. Clinical cases on the treatment of depression and anxiety have been frequently reported [18] [29]. Sufficient evidence shows that PPI is an effective approach to treat depression, anxiety, and other psycho- 
logical and psychiatric illness [30] [31].

Chen et al. [10] believed EWOT might share the same cultural origin with Morita Therapy and the skills of the two systems were similar. But differences existed between the procedures and appetencies. Morita therapy was regarded as the extension of EWOT in Chinese medicine psychology. The goals of Morita therapy are the recognition of facts, obedience to nature, focus on the present, the increase of spontaneous activities, the decrease of self-focused preoccupation, the elimination of indulgence in moods and emotions, the withholding of value judgments, the reduction of intellectualizing, the cessation of escape into a sick role, and the cultivation of a humble mind [32] [33]. The goal deeply rooted in Chinese Buddhism, Taoism and Confucianism [34].

Researchers compared EWOT with behavior therapy. Similarities were found in theoretic foundation and intervention intention, but the procedures, general protocol, and appetency were different [7] [12]. Taking HOS as example, behaviors such as jumping, dancing, howling, playing drum produce joyful emotion, which can decrease the disorders resulting from extreme sorrow or sadness. HOS is very similar with the positive reinforcement in behavior therapy. Positive reinforcement is applied to encourage and reward normal behaviors thus to suppress and replace bad behaviors [35].

\section{Conclusion}

In conclusion, EWOT is a unique therapeutic approach based on the emotion-will theory in Chinese medicine. Even though the conventional psychological modalities hold predominant status in China, it still requires localized adjustment to become more suitable for Chinese people. Although greatly influenced by western models, contemporary Chinese approaches to counseling reflect the philosophical traditions, cultural history, and indigenous help-seeking practices of a rapid modernizing society [36]. EWOT, rooted in Chinese culture and philosophy, ought to be more suitable without too big obstacles. Owing to the advantages of demanding simple environment, few disturbances, convenience, and compatibility with other Chinese medicine approaches, esp. acupuncture, EWOT is believed to have a promising prosperity and high value in dealing with psychological illness [37]. Disadvantages are unavoidable. Systemic therapeutic protocol has not been established. Lack of standards and effective evaluations remain a primary obstacle impeding the popularization and developing of EWOT. Our team and colleagues are trying to eliminate the obstacles in the following aspects: 1) designing rational trails and experiments to validate the efficacy and provide evidence for clinical use; 2) standardizing operation protocols; 3 ) developing assessment tools; 4) introducing EWOT abroad and studying it from cross-cultural perspective. We have strong faith in the bright future of this ancient, but new psychological therapy.

\section{Acknowledgements}

This study was under the support of National Science Funds of China (NSFC) with the Grant No. 81373710 and Science and Technology Development Fund of Chengdu University of TCM with the Grant No. (ZRMS-201348).

\section{Conflict of Interests}

The authors declare no conflict of interests.

\section{References}

[1] Shi, L. and Zhang, C. (2012) Spirituality in Traditional Chinese Medicine. Pastoral Psychology, 61, 959-974. http://dx.doi.org/10.1007/s11089-012-0480-x

[2] Longhurst, J.C. (2010) Defining Meridians: A Modern Basis of Understanding. Journal of Acupuncture and Meridian Studies, 3, 67-74. http://dx.doi.org/10.1016/S2005-2901(10)60014-3

[3] Dobos, G. and Tao, I. (2011) The Model of Western Integrative Medicine: The Role of Chinese Medicine. Chinese Journal of Integrative Medicine, 17, 11-20. http://dx.doi.org/10.1007/s11655-011-0601-x

[4] Pilkington, K. (2010) Anxiety, Depression and Acupuncture: A Review of the Clinical Research. Autonomic Neuroscience: Basic and Clinical, 157, 91-95. http://dx.doi.org/10.1016/j.autneu.2010.04.002

[5] Wang, J., Li, Y., Ni, C., Zhang, H., Li, L. and Wang, Q. (2011) Cognition Research and Constitutional Classification in Chinese Medicine. The American Journal of Chinese Medicine, 39, 651-660. http://dx.doi.org/10.1142/S0192415X11009093

[6] Xutian, S., Zhang, J. and Louise, W. (2009) New Exploration and Understanding of Traditional Chinese Medicine. The 
American Journal of Chinese Medicine, 37, 411-426. http://dx.doi.org/10.1142/S0192415X09006941

[7] Chen, R., Bi, Y., Qin. Z. and Yang, Y.Q. (2006) Research on Relationship between Behavior Therapy and Three Psychotherapy of TCM. J. Yunnan Univ. Tradit. Chin. Med., 29, 10-12, 21. (In Chinese)

[8] Huang, Z.B. (2009) Compared Positive Psychotherapy and Emotional Counterbalance Therapy. Chin. Med. Modern Distance Educ. China, 7, 84-85. (In Chinese)

[9] Aung, S.K. (1996) Medical Acupuncture and the Management of Psychosomatic Illness. Acupuncture in Medicine, 14, 84-88. http://dx.doi.org/10.1136/aim.14.2.84

[10] Chen, R., Qin, Z., Zhao, Z.Y., Yang, Y.Q., Chu, G.W., Yang, W.D. and Chen, P. (2004) The Research to the Relationship between the Therapy of Mutual Promotion and Restraint between the Five Elements and the Sentian Therapy. Chin. Arch. Tradit. Chin. Med., 22, 1218-1219. (In Chinese)

[11] Chan, C., Ying Ho, P.S. and Chow, E. (2002) A Body-Mind-Spirit Model in Health: An Eastern Approach. Social Work in Health Care, 34, 261-282. http://dx.doi.org/10.1300/J010v34n03 02

[12] Lin, K.M. (1981) Traditional Chinese Medical Beliefs and Their Relevance for Mental Illness and Psychiatry. Normal and Abnormal Behavior in Chinese Culture, 2, 95-111. http://dx.doi.org/10.1007/978-94-017-4986-2 6

[13] Sarris, J., Panossian, A., Schweitzer, I., Stough, C. and Scholey, A. (2011) Herbal Medicine for Depression, Anxiety and Insomnia: A Review of Psychopharmacology and Clinical Evidence. European Neuropsychopharmacology, 21, 841-860. http://dx.doi.org/10.1016/j.euroneuro.2011.04.002

[14] Tou, W.I., Chang, S.S., Lee, C.C. and Chen, C.Y.C. (2013) Drug Design for Neuropathic Pain Regulation from Traditional Chinese Medicine. Scientific Reports, 3, 844. http://dx.doi.org/10.1038/srep00844

[15] Ma, Y.X. (2010) The Chinese Medicine Sentiment Will Theory Source and Course Searches Analysis. Chinese Archives of Traditional Chinese Medicine, 28, 1838-1840. (In Chinese)

[16] Wang, X., Sun, H., Zhang, A., Sun, W., Wang, P. and Wang, Z. (2011) Potential Role of Metabolomics Approaches in the Area of Traditional Chinese Medicine: As Pillars of the Bridge between Chinese and Western Medicine. Journal of Pharmaceutical and Biomedical Analysis, 55, 859-868. http://dx.doi.org/10.1016/j.jpba.2011.01.042

[17] Wang, M.Q., Zou, Y.Z., Zheng, Q., Chen, C. and Tang, C.H. (2006) Analysis of 196 Cases in Ming Yi Lei An about Morbidity Construction and Male and Female Characteristics of Seven Modes of Emotion Injury. Modern J. Integrat. Tradit. Chin. West. Med., 15, 983-984. (In Chinese)

[18] Yang, S.M., Gong, X.H. and Hui, L.J. (2008) Discussion about Chinese Medical Therapeutics on Depression Insomnia. Lishizhen Medicine and Materia Medica Research, 19, 639-640. (In Chinese)

[19] Liu, Q. (2009) Clinical Use of Pensiveness Overcoming Fear. J. Hubei Univ. Chin. Med., 11, 52-53. (In Chinese)

[20] Kronenberg, F. and Fugh-Berman, A. (2002) Complementary and Alternative Medicine for Menopausal Symptoms: A Review of Randomized, Controlled Trials. Annals of Internal Medicine, 137, 805-813. http://dx.doi.org/10.7326/0003-4819-137-10-200211190-00009

[21] Nedrow, A., Miller, J., Walker, M., Nygren, P., Huffman, L.H. and Nelson, H.D. (2006) Complementary and Alternative Therapies for the Management of Menopause-Related Symptoms: A Systematic Evidence Review. Archives of Internal Medicine, 166, 1453-1465. http://dx.doi.org/10.1001/archinte.166.14.1453

[22] Zhang, H., Zhang, X.G., Liang, X.L., Wang, H.Y. and Liu, Y. (2014) Advance of Chinese Medicine Emotion Nursing. Chinese Journal of Convalescent Medicine, 23, 208-209. (In Chinese)

[23] Ye, L.X., Chen, X.L. and Jiang, Y.X. (2012) Impact of Emotion-Will Therapy on the Anxiety of Stroke Patients. Chin. J. Guangming Chin. Med., 27, 2462-2463. (In Chinese)

[24] Chen, X.L. (2012) Impact of Emotion-Will Therapy on the QoL of Bronchial Asthma. Chinese Journal of Modern Drug. 6, 118-119. (In Chinese)

[25] Liu, Q., Zhang, X.G., Liang, Q.F., Li, F.Y., Gao, J., Liang, X.L. and Yang, Y.Q. (2012) Expounding and Proving Favorable Environment and Earthquake Environment Model from SD Rat Offspring's Weight and Length. J. Liaoning Univ. Tradit. Chin. Med, 14, 82-84. (In Chinese)

[26] Zhang, X.G., Zhang, H., Tan, R., Peng, J.C., Liang, X.L., Liu, Q., Wang, M.Q. and Yu, X.P. (2012) Mechanism of Earthquake Simulation as a Prenatal Stressor Retarding Rat Offspring Development and Chinese Medicine Correcting the Retardation: Hormones and Gene-Expression Alteration. Evidence-Based Complementary and Alternative Medicine, 2012, Article ID: 670362. http://dx.doi.org/10.1155/2012/670362

[27] Yan, S.X., Zou, Y.Z., Cui, J.F. and Cao, Y.J. (2008) A Study of 122 Cases Analysis with Psychotherapy of Traditional Chinese Medicine. Lishizhen Medicine and Materia Medica Research, 19, 1471-1474. (In Chinese)

[28] Sin, N.L. and Lyubomirsky, S. (2009) Enhancing Well-Being and Alleviating Depressive Symptoms with Positive Psychology Interventions: A Practice-Friendly Meta-Analysis. Journal of Clinical Psychology, 65, 467-487.

http://dx.doi.org/10.1002/jclp.20593 
[29] Sun, Y. (2012) Approach of Application Classical Prescription in Treating Double Heart Disease. Tianjin Journal of Traditional Chinese Medicine, 29, 361-362.

[30] Manicavasagar, V., Horswood, D., Burckhardt, R., Lum, A., Hadzi-Pavlovic, D. and Parker, G. (2014) Feasibility and Effectiveness of a Web-Based Positive Psychology Program for Youth Mental Health: Randomized Controlled Trial. Journal of Medical Internet Research, 16, e140. http://dx.doi.org/10.2196/jmir.3176

[31] Schrank, B., Brownell, T., Tylee, A. and Slade, M. (2014) Positive Psychology: An Approach to Supporting Recovery in Mental Illness. East Asian Arch Psychiatry, 24, 95-103.

[32] Hwang, K.K. and Chang, J. (2009) Self-Cultivation Culturally Sensitive Psychotherapies in Confucian Societies. The Counseling Psychologist, 37, 1010-1032. http://dx.doi.org/10.1177/0011000009339976

[33] Gaudiano, B.A. (2009) Öst's (2008) Methodological Comparison of Clinical Trials of Acceptance and Commitment Therapy Versus Cognitive Behavior Therapy: Matching Apples with Oranges? Behaviour Research and Therapy, 47, 1066-1070. http://dx.doi.org/10.1016/j.brat.2009.07.020

[34] Qian, M., Smith, C.W., Chen, Z. and Xia, G. (2001) Psychotherapy in China: A Review of Its History and Contemporary Directions. International Journal of Mental Health, 30, 49-68.

[35] Kanter, J.W., Rusch, L.C., Busch, A.M. and Sedivy, S.K. (2009) Validation of the Behavioral Activation for Depression Scale (BADS) in a Community Sample with Elevated Depressive Symptoms. Journal of Behavior Therapy and Experimental Psychiatry, 31, 36-42. http://dx.doi.org/10.1007/s10862-008-9088-y

[36] Chang, D.F., Tong, H., Shi, Q. and Zeng, Q. (2005) Letting a Hundred Flowers Bloom: Counseling and Psychotherapy in the People's Republic of China. J. Mental Health Counseling, 27, 104-116.

[37] Gao, Z. (2013) The Strengths and Weaknesses of the Analysis of Emotion Therapy Clinical Applications. Inner Mongolia J. Chin. Med., 32, 140-141. (In Chinese) 